\title{
Berberine inhibits the MexXY-OprM efflux pump to reverse imipenem resistance in a clinical carbapenem-resistant Pseudomonas aeruginosa isolate in a planktonic state
}

\author{
FEN SU $^{1}$ and JILIANG WANG ${ }^{2}$ \\ ${ }^{1}$ Clinical Laboratory, Weifang People's Hospital, Weifang, Shandong $261041 ;{ }^{2}$ Clinical Laboratory, \\ Shengli Oilfield Central Hospital, Dongying, Shandong 257034, P.R. China
}

Received April 19, 2017; Accepted August 4, 2017

DOI: $10.3892 / \mathrm{etm} .2017 .5431$

\begin{abstract}
Pseudomonas (P.) aeruginosa is an ubiquitous and metabolically versatile opportunistic pathogen and may cause various life-threatening diseases. Due to increasing emergence of resistance to carbapenems, novel drugs with improved antibacterial activities compared with those of traditional antibiotics are required. In the present study, berberine (BEB), a natural isoquinoline alkaloid, was used in combination with imipenem (IMP), a commonly-used carbapenem, to investigate their antibacterial activities against a clinical $P$. aeruginosa isolate PA012 and the potential mechanism. Screening revealed that the minimum inhibitory concentrations (MICs) of BEB and IMP were 512 and $256 \mu \mathrm{g} / \mathrm{ml}$, respectively. The combination of BEB $(1 / 4$ MIC) and IMP (1/8 MIC) exhibited a synergistic effect with a fractional inhibitory concentration index of 0.375 . The synergism of BEB and IMP was also demonstrated in a time-kill test and by scanning electron microscopic observation. Treatment with BEB at $1 / 4$ MIC in combination with IMP at $1 / 16,1 / 8,1 / 4$ and $1 / 2$ MIC revealed a concentration-dependent promoting effect of IMP on the intracellular accumulation of BEB and inhibition of bacterial adhesion. Further analysis of gene expression revealed that BEB (1/4 MIC) combined with IMP (1/8 MIC) decreased MexZ, MexX, MexY and outer membrane protein (Opr)M by 38, 35, 46 and $49 \%$ in PA012. In conclusion, these results suggested that IMP had synergistic effects with BEB against the clinical isolate PA012 via inhibition of the MexXY-OprM efflux pump.
\end{abstract}

Correspondence to: Dr Jiliang Wang, Clinical Laboratory, Shengli Oilfield Central Hospital, 31 Jinan Road, Dongying, Shandong 257034, P.R. China

E-mail: wangj117@126.com

Key words: berberine, imipenem, synergism, Pseudomonas aeruginosa, efflux pump, MexXY-OprM

\section{Introduction}

Pseudomonas (P.) aeruginosa is a ubiquitous and metabolically versatile opportunistic pathogen, which may colonize in the respiratory tract/gastrointestinal mucosa of patients as well as medical equipment, including catheters and intubation tubes. This Gram-negative bacterium may cause a series of infectious diseases, including keratitis, otitis media and bacteremia, in immuno-compromised patients and those in intensive care units with high morbidity and mortality (1). Due to extensive application of antibiotics, an increasing number of $P$. aeruginosa isolates have been identified to be resistant to various traditional antibacterial agents, including penicillins, fluoroquinolones, aminoglycosides, polymyxins as well as carbapenems, via intrinsic as well as acquired resistance mechanisms (1). One of the essential factors contributing to resistance is associated with efflux pumps, of which four types, namely MexAB-outer membrane protein (Opr)M, MexEF-OprN, MexCD-OprJ and MexXY-OprM, have been widely studied (2). Kiser et al (3) reported that OprM and a group of efflux regulatory genes or efflux protein (NfxB, MexR and MexZ) contributed to multidrug resistance in clinical $P$. aeruginosa isolates.

Although MexAB-OprM is thought to be mostly accountable for carbapenem resistance in $P$. aeruginosa rather than MexXY-OprM (4,5), increasing evidence indicated that MexXY-OprM was also inclined to be overexpressed in clinical P. aeruginosa isolates. In A Brazilian study by Xavier et al (6) demonstrated that MexXY-OprM was overexpressed in $50.8 \%$ of $P$. aeruginosa isolates studied compared with $27.1 \%$ for MexAB-OprM. A study from Thailand by Khuntayaporn et al (7) observed that, increased expression of MexAB-OprM mRNA accounted for $92.06 \%$ followed by $63.49 \%$ for Mex XY-OprM mRNA. Obviously, the percentages of MexAB-OprM and MexXY-OprM overexpression exhibited geographical variations.

Imipenem (IMP), a carbapenem, has an important role in the treatment of infections caused by multidrug-resistant $P$. aeruginosa. However, it has been reported that $20 \%$ of $P$. aeruginosa isolates were resistant to IMP (8). Due to this IMP resistance in $P$. aeruginosa novel antibacterial agents are urgently required. Berberine (BEB), a natural isoquinoline alkaloid, is prevalent in numerous medicinal plants and 
has been demonstrated to have antibacterial and antifungal activities alone or in combination with other drugs (9-13). In a previous study, BEB was reported to be a potential inhibitor of MexXY-mediated resistance in P. aeruginosa (14). However, the antipseudomonas activity of BEB in combination with other traditional antibiotics, including IMP, in the treatment of clinical $P$. aeruginosa isolates has remained largely elusive.

The present study aimed to investigate the efficacy of BEB alone and in combination with IMP against a clinical $P$. aeruginosa isolate and assessed the underlying mechanism from the viewpoint of the MexXY-OprM efflux pump.

\section{Materials and methods}

Bacterial strains. The $P$. aeruginosa ATCC27853 strain was obtained from the National Institute for the Control of Pharmaceutical and Biological Products (Beijing, China). The clinical isolate, PA012, was isolated from a patient with a urinary tract infection at and provided by the Clinical Laboratory of Weifang People's Hospital (Weifang, China). The present study was approved by the ethics committee of the Weifang People's Hospital. Written informed consent was obtained from the patient prior to the collection of PA012. The strains were stored at $-80^{\circ} \mathrm{C}$ and subjected to cultivation in Luria-Bertani medium (Hope, Qingdao, China) with constant shaking at $8 \mathrm{x} \mathrm{g}$ at $37^{\circ} \mathrm{C}$ for $18 \mathrm{~h}$ until the strains reached the late logarithmic growth phase.

Susceptibility assay. The minimum inhibitory concentrations (MICs) of BEB and IMP were assessed using the broth microdilution method following the criteria of the Clinical and Laboratory Standards Institute (no. M07-A10) (15). The bacterial suspension was adjusted to $1 \times 10^{5}$ colony-forming units $(\mathrm{CFU}) / \mathrm{ml}$ and incubated with BEB/IMP in a $96-$ well flat-bottomed microtiter plate for $24 \mathrm{~h}$ at $35^{\circ} \mathrm{C}$. The concentrations of BEB/IMP were serially two-fold diluted in a range of $2-1,014 \mu \mathrm{g} / \mathrm{ml}$ for BEB and $0.25-128 \mu \mathrm{g} / \mathrm{ml}$ for IMP. The MIC was defined as the lowest concentration of BEB/IMP to cause no visual growth of bacteria. The combination of BEB and IMP was evaluated by checkerboard assay. The concentrations of BEB and IMP were set in a range of 1/64-1 MIC for BEB and 1/64-16 MIC for IMP. The fractional inhibitory concentration index (FICI) was determined using the following equation: $\mathrm{FICI}=\left(\mathrm{MIC}_{\mathrm{BEB}}\right.$ in combination $/ \mathrm{MIC}_{\mathrm{BEB}}$ alone $)+\left(\mathrm{MIC}_{\mathrm{IMP}}\right.$ in combination/ $\mathrm{MIC}_{\mathrm{IMP}}$ alone), in which synergism, indifference and antagonism were interpreted as FICI $\leq 0.5,>0.5$ and $\leq 4.0$, and $>4.0$, respectively (16).

Time-kill (T-K) test. The initial PA012 suspension $\left(1 \times 10^{5} \mathrm{CFU} / \mathrm{ml}\right)$ was incubated with BEB and/or IMP at their concentrations at which synergism had been previously determined by using the checkerboard assay (17). The cells were counted at the designated time-points $(0,4,8,12$ and $24 \mathrm{~h})$. The control group remained untreated. T-K curves were plotted on a logarithmic scale of CFU/ml vs. time (h). Synergism was defined as an increase of $\geq 2 \log 10 \mathrm{CFU} / \mathrm{ml}$ in the killing rate for the combined agents compared with the most active agent used alone. Antagonism was defined as a decrease of $\geq 2 \log 10 \mathrm{CFU} / \mathrm{ml}$ in the killing rate for the combined agents compared with the most active agent alone. Indifference was defined as a decrease of $\leq 2 \log 10 \mathrm{CFU} / \mathrm{ml}$ in the killing rate for the combined agents compared with any of the two agents used alone (17).

Scanning electron microscopy (SEM). Prior to treatments with BEB (1/4 MIC) and/or IMP (1/8 MIC), a suspension of PA012 $\left(100 \mu \mathrm{l} ; 1 \times 10^{5} \mathrm{CFU} / \mathrm{ml}\right)$ was added into a 6 -well flat-bottomed microtiter plate with sterile cover slips, followed by incubation for $24 \mathrm{~h}$. The cover slips were then rinsed with sterile PBS, fixed with $2.5 \%$ (v/v) glutaraldehyde overnight at room temperature, and post-fixed with $0.1 \%$ osmium tetroxide for $1 \mathrm{~h}$. The samples were subsequently dehydrated with a series of $30,50,70,90$ and $100 \%$ ethanol for 10 min each. After critical-point drying, the samples were processed by gold sputtering (JEOL JFC 1200E Ion sputtering device; JEOL Ltd., Tokyo, Japan) and observed by SEM (JSM-6700F; JEOL Ltd.).

Adhesion test. PA012 suspension $\left(100 \mu \mathrm{l} ; 1 \times 10^{5} \mathrm{CFU} / \mathrm{ml}\right)$ was incubated with BEB (1/4 MIC) in combination with IMP at $1 / 16,1 / 8,1 / 4$ and $1 / 2 \mathrm{MIC}$ for 2 or $4 \mathrm{~h}$. The adhesion test was performed on the surface of medical-grade polyvinyl chloride (Shengli Oilfield Central Hospital, Dongying, China). The medium was discarded and the wells were washed with $\mathrm{pH} 7.2$ sterile PBS. The bacterial cells in each well were then stained with $150 \mu \mathrm{l} 0.1 \%$ crystal violet for $15 \mathrm{~min}$ at room temperature. Following washing with PBS for several times and drying for $3 \mathrm{~h}, 150 \mu \mathrm{l} 30 \%$ (v/v) acetic acid was added to the stained cells, followed by incubation with mixing for $15 \mathrm{~min}$. The optical density at the wavelength of $492 \mathrm{~nm}\left(\mathrm{OD}_{492}\right)$ was measured.

BEB uptake. PA012 cells in the late logarithmic growth phase were centrifuged at $10,000 \mathrm{x}$ g for $5 \mathrm{~min}$ at $35^{\circ} \mathrm{C}$, washed twice with $0.01 \mathrm{M}$ sterile PBS (pH 7.2) and resuspended in $1 \mathrm{ml}$ PBS to reach an $\mathrm{OD}_{600}$ of 0.2 . The cells were then treated with $\mathrm{BEB}$ (1/4 MIC) or in combination with IMP at 1/16, 1/8, 1/4 and 1/2 of the MIC at $37^{\circ} \mathrm{C}$ for $10 \mathrm{~min}$. Cells killed in a $100^{\circ} \mathrm{C}$ water bath were incubated with the same concentrations of agents were set as the control. Subsequently, the cells were centrifuged, washed and resuspended to reach an $\mathrm{OD}_{600}$ of 0.15 . The fluorescence of BEB was determined with a Synergy ${ }^{\mathrm{TM}}$ HT multidetection microplate reader (BioTek, Winooski, VT, USA) at $360 \mathrm{~nm}$ excitation wavelength and $530 \mathrm{~nm}$ emission wavelength (18).

Reverse-transcription quantitative polymerase chain reaction (RT-qPCR) analysis. The PA012 cells $\left(1 \times 10^{5} \mathrm{CFU} / \mathrm{ml}\right)$ were first treated with BEB (1/4 MIC) and/or IMP (1/8 MIC). The total RNA was then extracted according to the instructions of the MagExtractor-RNA-kit (cat. no. NPK-200; Toyobo Life Science, Osaka, Japan). Complementary DNA was prepared following the instructions of the ReverTra Ace ${ }^{\circledR}$ qPCR RT Master Mix with gDNA Remover kit (cat. no. FSQ-301; Toyobo Life Science). Primers with the following sequences were used: MexZ forward, 5'-CCCTTGTGAGGACGTTCA GT-3' and reverse, 5'-CCAGCAACAGGTAGGGAGAA-3'; MexX forward, 5'-CATCAGCGAACGCGAGTACAC-3' and reverse, 5'-CAATTCGCGATGCGGATTG-3'; MexY forward 5'-CCGCTACAACGGCTATCCCT-3' and reverse, 5'-AGC GGGATCGACCAGCTTTC-3'; OprM forward 5'-GATCCC CGACTACCAGCGCCCCG-3' and reverse, 5'-ATGCGGTAC 
Table I. Interaction of BEB and IMP alone and in combination against Pseudomonas aeruginosa.

\begin{tabular}{|c|c|c|c|c|c|}
\hline \multirow[b]{2}{*}{ Strain } & \multicolumn{2}{|c|}{$\begin{array}{l}\text { MIC alone } \\
(\mu \mathrm{g} / \mathrm{ml})\end{array}$} & \multicolumn{2}{|c|}{$\begin{array}{l}\text { MIC in combination } \\
(\mu \mathrm{g} / \mathrm{ml})\end{array}$} & \multirow[b]{2}{*}{ FICI (interpretation) } \\
\hline & BEB & IMP & BEB & IMP & \\
\hline PA ATCC27853 & 512 & 1 & 256 & 1 & 1.5 (indifference) \\
\hline PA012 & 512 & 256 & 128 & 32 & 0.375 (synergism) \\
\hline
\end{tabular}

PA, Pseudomonas aeruginosa; ATCC, American Type Tissue Collection; MIC, minimum inhibitory concentration; BEB, berberine; IMP, imipenem; FICI, fractional inhibitory concentration index.

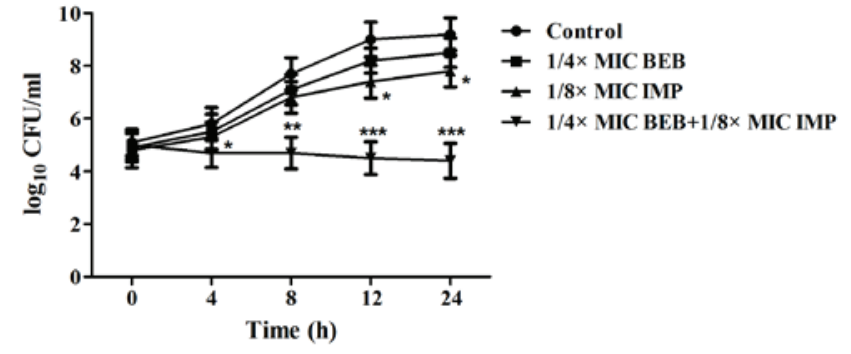

Figure 1. T-K curves of PA012 cells $\left(1 \times 10^{5} \mathrm{CFU} / \mathrm{ml}\right)$ treated with or without 1/4 MIC BEB and/or 1/8 MIC IMP. The cells were counted at each time-point $(0,4,8,12$ and $24 \mathrm{~h})$. The T-K curves were plotted on a logarithmic scale as $\mathrm{CFU} / \mathrm{ml}$ vs. time (h). The control group had no agents. ${ }^{*} \mathrm{P}<0.05 ;{ }^{* *} \mathrm{P}<0.01$; ${ }^{* * * *} \mathrm{P}<0.001$ vs. the control group. CFU, colony-forming units; MIC, minimum inhibitory concentration; BEB, berberine; IMP, imipenem; T-K, Time-Kill.

TGCGCCCGGAAGGC-3'; OprD forward 5'-ATCTACCGC ACAAACGATGAG-3' and reverse, 5'-GCCGAAGCCGAT ATAATCAAACG-3'; RpsL forward 5'-GCAAGCGCATGG TCGACAAGA-3' and reverse, 5'-CGCTGTGCTCTTGCA GGTTGTGA-3'. The $25 \mu \mathrm{l}$ qPCR mixture consisted of $12.5 \mu \mathrm{l}$ 2X SYBR ${ }^{\circledR}$ Green Real Time PCR Master mix, $1 \mu$ l PCR forward primer, $1 \mu \mathrm{l}$ PCR reverse primer, $0.5 \mu \mathrm{l} \mathrm{cDNA}$ and $10 \mu \mathrm{l}$ double distilled water. RT-qPCR was performed on an ABI7000 fluorescent qPCR system (Thermo Fisher Scientific, Inc., Waltham, MA, USA). The thermocycling steps were as follows: $95^{\circ} \mathrm{C}$ for $10 \mathrm{~min}, 40$ cycles of $95^{\circ} \mathrm{C}$ for $15 \mathrm{sec}, 55^{\circ} \mathrm{C}$ for $10 \mathrm{sec}$ and $72^{\circ} \mathrm{C}$ for $10 \mathrm{sec}$, and final elongation at $72^{\circ} \mathrm{C}$ for $5 \mathrm{~min}$. All data were normalized to reference gene RpsL. The relative target-gene expression was calculated using the $2^{-\Delta \Delta C q}$ method (19).

Statistical analysis. Results were obtained from three independent experiments performed in triplicate. Values are expressed as the mean \pm standard deviation. Statistical analysis was performed using SPSS 19.0 software (IBM Corp., Armonk, NY, USA). Differences between groups were determined using one-way analysis of variance with a Student-Newman-Keuls method post hoc test. $\mathrm{P}<0.05$ was considered to indicate a statistically significant difference.

\section{Results}

Synergistic effect of BEB and IMP against PA012. The MIC of BEB was $512 \mu \mathrm{g} / \mathrm{ml}$ in PA ATCC27853 as well as in PA012 cells, displaying weak antibacterial activity. Although the MIC of BEB in PA ATCC27853 cells was decreased 2-fold if used in combination with IMP at its MIC of $1 \mu \mathrm{g} / \mathrm{ml}$, it was not synergistic with IMP, as the FICI was 1.5 , which was interpreted as being indifferent. In the clinical isolate, PA012, the MIC of IMP was $265 \mu \mathrm{g} / \mathrm{ml}$, and a synergism of BEB and IMP was identified with an FICI of $0.375(<0.5)$. The MIC of BEB and IMP declined 4-and 8-fold, respectively, in combination compared with each alone (Table I). The synergism of BEB and IMP in PA012 cells was also evaluated by a T-K test. The results indicated that BEB (1/4 MIC) plus IMP (1/8 MIC) or IMP (1/8 MIC) alone significantly reduced the quantities of PA012 ( $\mathrm{P}<0.05$ vs. the control group), but the growth continued. The combination of BEB and IMP completely inhibited the bacterial growth, with a decrease of $3.4 \log _{10}$ $\mathrm{CFU} / \mathrm{ml}$ compared with IMP, the most effective agent, alone (Fig. 1). Furthermore, the synergistic activity of BEB with IMP was confirmed by SEM evaluation, displaying a marked decrease in the density of PA012 cells compared with that in the control group (Fig. 2).

$B E B$ and IMP have synergistic effects via enhancement of intracellular BEB accumulation and inhibition of cell adherence. In the subsequent experiments, the concentration of BEB was set at $1 / 4$ of the MIC to investigate the effects of its combination with a series of IMP at concentrations of $1 / 16$, $1 / 8,1 / 4$ and $1 / 2$ of the MIC on bacterial adhesion and BEB uptake into PA012 cells. It was observed that BEB (1/4 MIC) plus IMP (1/16 MIC) did not produce any evident inhibition of the bacterial adhesion at neither 2 nor $4 \mathrm{~h}$, while application of BEB (1/4 MIC) plus IMP at $1 / 8,1 / 4$ and $1 / 2$ of the MIC resulted in a marked decrease of bacterial adhesion at $2 \mathrm{~h}$ $(\mathrm{P}<0.05, \mathrm{P}<0.05$ and $\mathrm{P}<0.01$, respectively) and at $4 \mathrm{~h}(\mathrm{P}<0.05$, $\mathrm{P}<0.01$ and $\mathrm{P}<0.01$, respectively; Fig. 3). BEB has fluorescent properties that may utilized for quantifying its intracellular accumulation. While significant fluorescence was detected in the BEB only group compared with the control, IMP significantly increased the fluorescence of BEB $(\mathrm{P}<0.01$ or $\mathrm{P}<0.001$; Fig. 4).

$B E B$ and IMP synergistically inhibit the expression of MexXY-associated genes. As demonstrated above, increasing concentrations of IMP promoted the intracellular accumulation of BEB in PA012. RT-qPCR was then used to analyze the antibacterial effects of BEB and IMP alone and in combination on the genes associated with the MexXY-OprM efflux pump in PA012. Compared with the control, BEB at 1/4 of the 

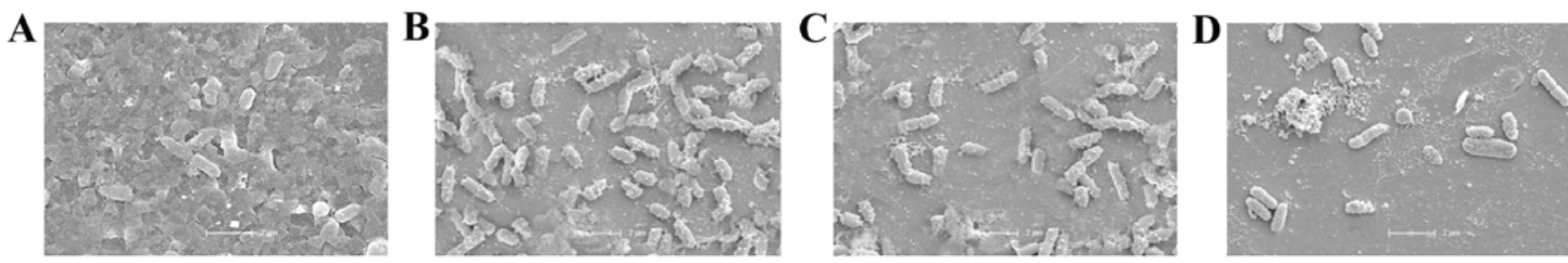

Figure 2. Scanning electron microscopy images of PA012 after incubation with (A) no drug, (B) BEB (1/4 MIC), (C) IMP (1/8 MIC) and (D) BEB (1/4 MIC) + IMP (1/8 MIC). Scale bar, $2 \mu \mathrm{m}$. MIC, minimum inhibitory concentration; BEB, berberine; IMP, imipenem.

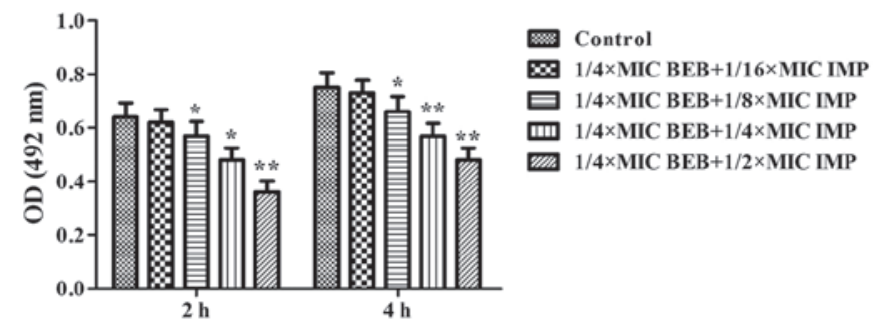

Figure 3. Bacterial adhesion of PA012 was evaluated by crystal violet staining after treatment with BEB (1/4 MIC) in combination with increasing concentrations of IMP $(1 / 16,1 / 8,1 / 4$ and $1 / 2 \mathrm{MIC})$ for 2 or $4 \mathrm{~h}$ and detection of the OD at $492 \mathrm{~nm} .{ }^{*} \mathrm{P}<0.05 ;{ }^{* *} \mathrm{P}<0.01$ vs. the control group. OD, optical density; MIC, minimum inhibitory concentration; BEB, berberine; IMP, imipenem.

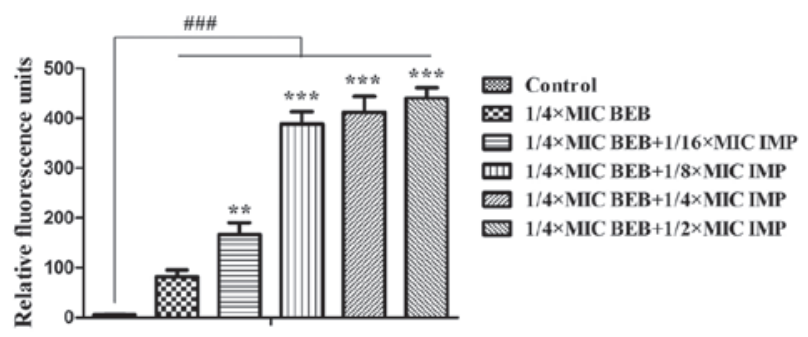

Figure 4. Relative fluorescence units were measured to evaluate BEB uptake in PA012 after treatment with BEB (1/4 MIC) in combination with increasing concentrations of IMP $(1 / 16,1 / 8,1 / 4$ and 1/2 MIC) at the excitation wavelength of $360 \mathrm{~nm}$ and the emission wavelength of $530 \mathrm{~nm}$. ${ }^{\# \#} \mathrm{P}<0.001 \mathrm{vs}$. the control group; ${ }^{* *} \mathrm{P}<0.01 ;{ }^{* * *} \mathrm{P}<0.001$ compared with the cells treated with BEB at 1/4 MIC. MIC, minimum inhibitory concentration; BEB, berberine; IMP, imipenem.

MIC and IMP at $1 / 8$ of the MIC alone inhibited the expression of MexZ $(\mathrm{P}<0.05)$, MexX $(\mathrm{P}<0.05)$, MexY $(\mathrm{P}<0.01)$ and $\mathrm{OprM}$ $(\mathrm{P}<0.05, \mathrm{P}<0.01)$. Of note, BEB plus IMP at the abovementioned concentrations resulted in decreases of $38 \%$ for MexZ $(\mathrm{P}<0.01), 35 \%$ for $\operatorname{Mex} \mathrm{X}(\mathrm{P}<0.01), 46 \%$ for MexY $(\mathrm{P}<0.001)$ and $49 \%$ for OprM $(\mathrm{P}<0.001)$. However, OprD expression was not significantly affected by treatment with BEB and/or IMP (Fig. 5A). It was also demonstrated that the expression of OprD in PA012 was identical to that in PA ATCC27853, thereby excluding the possibility of OprD loss in response to the treatments (Fig. 5B).

\section{Discussion}

Increasing carbapenem resistance in clinical $P$. aeruginosa isolates caused by IMP is responsible for the occurrence of life-threatening nosocomial diseases. The present study demonstrated for the first time, to the best of our knowledge, that BEB was able to enhance the antibacterial effect of IMP in a clinical $P$. aeruginosa isolate by using a susceptibility test, T-K assay, adhesion measurement and SEM evaluation. Furthermore, it was demonstrated that the reversion of IMP resistance by BEB may be due to inhibition of the MexXY-OprM efflux pump.

Among the four efflux pumps associated with carbapenem resistance, MexAB-OprM and MexXY-OprM are constitutively expressed and their overexpression mediates intrinsic resistance in P. aeruginosa, while MexCD-OprJ and MexEF-OprN usually remain quiescent in wild-type $P$. aeruginosa and their overexpression results in acquired multi-drug resistant phenotypes in mutant isolates (20-22). As described previously, MexAB-OprM and MexXY-OprM are usually overexpressed in clinical $P$. aeruginosa isolates $(4,5)$, and the latter was also demonstrated to be a target of BEB (14). Therefore, MexXY-OprM was extensively investigated in the present study.

The antipseudomonas activity of BEB has been revealed to be rather weak (MIC $\geq 512 \mu \mathrm{g} / \mathrm{ml})(14,17)$. In accordance with this, the present study also found a relatively high MIC of BEB in P. aeruginos $a$. However, BEB combined with IMP exhibited strong synergism, inferring the potential of BEB to reverse IMP resistance or enhance the susceptibility of $P$. aeruginosa to IMP. PCR analysis also demonstrated that BEP plus IMP significantly decreased the mRNA expression of MexX, MexY and MexZ, which was responsible for MexXY-OprM repression, while the inhibitory effect of BEB alone on the expression of the three genes was less pronounced. Sub-MIC IMP has been reported to possess antipseudomonas activity (23). As is known, the major mechanism of resistance to IMP may be attributed to the loss or mutation of $\mathrm{OprD}$, which is primarily in charge of basic amino acid uptake and transport of carbapenems across the outer membrane (4). Of note, the present study did not observe any significant changes in OprD mRNA in the clinical P. aeruginosa isolates following incubation with BEB and/or IMP. Compared with the standard $P$. aeruginosa strain PA ATCC27853, an equivalent expression of OprD mRNA was noted in the clinical isolate PA012, indicating no loss of the OprD gene. Due to experimental restrictions, it was not determined whether the OprD gene was mutated in the clinical $P$. aeruginosa isolate. The present results implied that BEB has a synergistic effect with IMP by inhibiting the MexXY-OprM efflux pump system; however, the roles of OprD in the effects of the drug combination require further evaluation. Distinct 
A

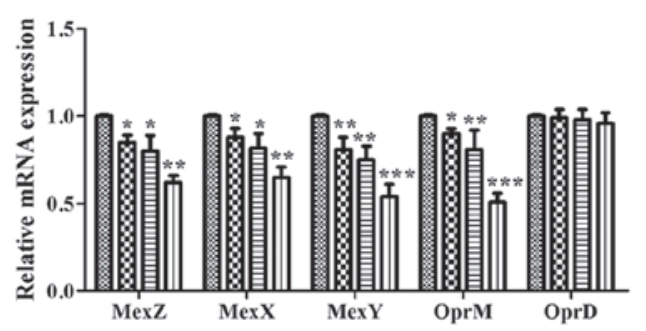

B

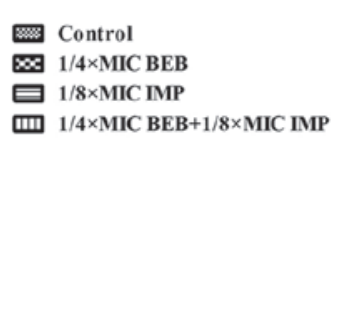

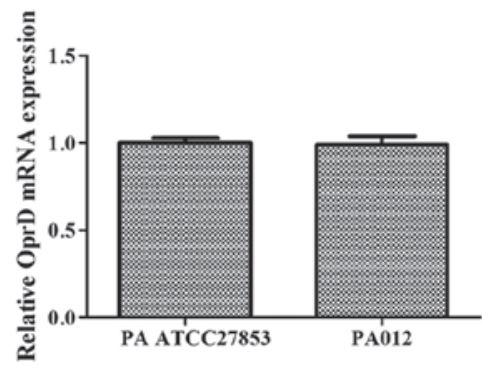

Figure 5. mRNA expression of (A) MexZ, MexX, MexY, OprM and OprD in PA012 treated with or without BEB (1/4 MIC) and/or IMP (1/8 MIC) and (B) OprD in PA ATCC27853 and PA012 without antibacterial agent treatments was analyzed by reverse-transcription quantitative polymerase chain reaction. RpsL was used as the reference gene. ${ }^{*} \mathrm{P}<0.05 ;{ }^{* *} \mathrm{P}<0.01 ;{ }^{* * * *} \mathrm{P}<0.001$ vs. the control group. MIC, minimum inhibitory concentration; BEB, berberine; IMP, imipenem; $\mathrm{PA}$, Pseudomonas aeruginosa; ATCC, American Type Tissue Collection; Opr, outer membrane protein.

from the conventional concept, El Amin et al (24) reported that alterations of OprD and efflux pumps may in part explain for carbapenem resistance patterns in clinical $P$. aeruginosa isolates. The present study did not assess all efflux pump systems, so that the possible participation of efflux pumps other than MexXY-OprM cannot be ruled out.

Several studies indicated that the weak antibacterial effect of BBR was mainly due to the low accumulation of intracellular BBR, and drugs that increased the intracellular BBR concentration were able to promote the antibacterial effect of BBR (25-28). The present study observed that IMP significantly increased the intracellular BBR fluorescence. BER has also been reported to exert its effects via targeting the cytoplasmic membrane and intra-nuclear DNA $(18,29)$. Collectively, based on the present results, it may be speculated that IMP had synergistic effects with BEB via increasing the accumulation of BEB in the intracellular space of $P$. aeruginosa cells. However, whether the ingressive BEB finally caused cell cycle arrest and DNA damage similar to its effects in Candida albicans still requires to be elucidated (13).

In conclusion, the present study suggested that BEB has a synergistic effect with IMP to re-sensitize IMP-resistant $P$. aeruginosa via inhibiting the MexXY-OprM efflux pump system. In future studies, more clinical $P$. aeruginosa isolates will be collected to confirm the synergistic activity of BEB and IMP, and simultaneously, nucleotide sequences of OprD should be analyzed. The antibacterial effects of BEB and/or IMP will also be evaluated in in vivo studies and relevant experiments are underway.

\section{References}

1. Morita Y, Tomida J and Kawamura Y: Responses of Pseudomonas aeruginosa to antimicrobials. Front Microbiol 4: 422, 2014.

2. Li XZ, Plésiat P and Nikaido H: The challenge of efflux-mediated antibiotic resistance in Gram-negative bacteria. Clin Microbiol Rev 28: 337-418, 2015.

3. Kiser TH, Obritsch MD, Jung R, MacLaren R and Fish DN: Efflux pump contribution to multidrug resistance in clinical isolates of Pseudomonas aeruginosa. Pharmacotherapy 30: 632-638, 2010.

4. Livermore DM: Of Pseudomonas, porins, pumps and carbapenems. J Antimicrob Chemother 47: 247-250, 2001.

5. Okamoto K, Gotoh $\mathrm{N}$ and Nishino T: Extrusion of penem antibiotics by multicomponent efflux systems MexAB-OprM, MexCD-OprJ and MexXY-OprM of Pseudomonas aeruginosa. Antimicrob Agents Chemother 46: 2696-2699, 2002.
6. Xavier DE, Picão RC, Girardello R, Fehlberg LC and Gales AC: Efflux pumps expression and its association with porin down-regulation and beta-lactamase production among Pseudomonas aeruginosa causing bloodstream infections in Brazil. BMC Microbiol 10: 217, 2010.

7. Khuntayaporn P, Montakantikul P, Santanirand P, Kiratisin P and Chomnawang MT: Molecular investigation of carbapenem resistance among multidrug-resistant Pseudomonas aeruginosa isolated clinically in Thailand. Microbiol Immunol 57: 170-178, 2013.

8. American Thoracic Society; Infectious Diseases Society of America: Guidelines for the management of adults with hospital-acquired, ventilator-associated andhealthcare-associated pneumonia. Am J Respir Crit Care Med 171: 388-416, 2005.

9. Guo N,Zhao X, Li W, Shi C, Meng R, Liu Z and Yu L: The synergy of berberine chloride and totarol against Staphylococcus aureus grown in planktonic and biofilm cultures. J Med Microbiol 64: 891-900, 2015.

10. Dziedzic A, Wojtyczka RD and Kubina R: Inhibition of oral Streptococci growth induced by the complementary action of berberine chloride and antibacterial compounds. Molecules 20: 13705-13724, 2015.

11. Budeyri Gokgoz N, Avci FG, Yoneten KK, Alaybeyoglu B, Ozkirimli E, Sayar NA, Kazan D and Sariyar Akbulut B: Response of Escherichia coli to prolonged berberine exposure. Microb Drug Resist 23: 531-544, 2017.

12. Shao J, Shi G, Wang T, Wu D and Wang C: Antiproliferation of berberine in combination with fluconazole from the perspectives of reactive oxygen species, ergosterol and drug efflux in a fluconazole-resistant Candida tropicalis isolate. Front Microbiol 7: $1516,2016$.

13. Li D, Xu Y, Zhang D, Quan H, Mylonakis E, Hu D, Li M, Zhao L, Zhu L, Wang Y and Jiang YY: Fluconazole assists berberine to kill fluconazole-resistant Candida albicans. Antimicrob Agents Chemother 57: 6016-6027, 2013.

14. Morita Y, Nakashima K, Nishino K, Kotani K, Tomida J, Inoue M and Kawamura Y: Berberine is a novel type efflux inhibitor which attenuates the MexXY-mediated aminoglycoside resistance in Pseudomonas aeruginosa. Front Microbiol 7: 1223, 2016.

15. Clinical and Laboratory Standards Institute (CLSI): Methods for dilution antimicrobial susceptibility tests for bacteria that grow aerobically; Approved Standard. CLSI Document M07-A10. 10th edition. CLSI, Wayne, PA, USA, 2015.

16. Odds F: Synergy, antagonism and what the chequerboard puts between them. J Antimicrob Chemother 52: 1, 2003.

17. Jayaraman $\mathrm{P}$, Sakharkar MK, Lim CS, Tang TH and Sakharkar KR: Activity and interactions of antibiotic and phytochemical combinations against Pseudomonas aeruginosa in vitro. Int J Biol Sci 6: 556-568, 2010.

18. Wang X, Yao X, Zhu Z, Tang T, Dai K, Sadovskaya I, Flahaut S and Jabbouri S: Effect of berberine on Staphylococcus epidermidis biofilm formation. Int J Antimicrob Agents 34: 60-66, 2009.

19. Livak KJ and Schmittgen TD: Analysis of relative gene expression data using real-time quantitative PCR and the 2(-Delta Delta C(T)) method. Methods 25: 402-408, 2001.

20. Poole K and Srikumar R: Multidrug efflux in Pseudomonas aeruginosa: Components, mechanisms and clinical significance. Curr Top Med Chem 1: 59-71, 2001. 
21. Sobel ML, Hocquet D, Cao L, Plesiat P and Poole K: Mutations in PA3574 (nalD) lead to increased MexAB-OprM expression and multidrug resistance in laboratory and clinical isolates of Pseudomonas aeruginosa. Antimicrob Agents Chemother 49: 1782-1786, 2005

22. Cao L, Srikumar R and Poole K: MexAB-OprM hyperexpression in NalC-type multidrug-resistant Pseudomonas aeruginosa: Identification and characterization of the nalC gene encoding a repressor of PA3720-PA3719. Mol Microbiol 53: 1423-1436, 2004.

23. Fonseca AP and Sousa JC: Effect of antibiotic-induced morphological changes on surface properties, motility and adhesion of nosocomial Pseudomonas aeruginosa strains under different physiological states. J Appl Microbiol 103: 1828-1837, 2007.

24. El Amin N, Giske CG, Jalal S, Keijser B, Kronvall G and Wretlind B: Carbapenem resistance mechanisms in Pseudomonas aeruginosa: Alterations of porin OprD and efflux proteins do not fully explain resistance patterns observed in clinical isolates. APMIS 113: 187-196, 2005

25. Stermitz FR, Lorenz P, Tawara JN, Zenewicz LA and Lewis K: Synergy in a medicinal plant: Antimicrobial action of berberine potentiated by 5'-methoxyhydnocarpin, a multidrug pump inhibitor. Proc Natl Acad Sci USA 97: 1433-1437, 2000 .
26. Ettefagh KA, Burns JT, Junio HA, Kaatz GW and Cech NB: Goldenseal (Hydrastis canadensis L.) Extracts synergistically enhance the antibacterial activity of berberine via efflux pump inhibition. Planta Med 77: 835-840, 2011.

27. Ball AR, Casadei G, Samosorn S, Bremner JB, Ausubel FM, Moy TI and Lewis K: Conjugating berberine to a multidrug efflux pump inhibitor creates an effective antimicrobial. ACS Chem Biol 1: 594-600, 2006.

28. Samosorn S, Tanwirat B, Muhamad N, Casadei G, Tomkiewicz D, Lewis K, Suksamrarn A, Prammananan T, Gornall KC, Beck JL and Bremner JB: Antibacterial activity of berberine-NorA pump inhibitor hybrids with a methylene ether linking group. Bioorg Med Chem 17: 3866-3872, 2009.

29. Severina II, Muntyan MS, Lewis K and Skulachev VP: Transfer of cationic antibacterial agents berberine, palmatine and benzalkonium through bimolecular planar phospholipid film and Staphylococcus aureus membrane. IUBMB Life 52: 321-324, 2001. 